\title{
Two-Way Relay Selection in Multiple Relayed FSO Networks
}

\author{
Parul Puri, Student Member, IEEE, Nestor D. Chatzidiamantis, Member, IEEE, Parul Garg, Senior Member, IEEE, \\ Mona Aggarwal, Student Member, IEEE, and George K. Karagiannidis, Fellow, IEEE
}

\begin{abstract}
In this letter, a parallel relayed free-space optical (FSO) network is considered. The network consists of multiple half-duplex amplify-and-forward two-way relays (TWRs), that assist in establishing communication between two distant nodes. A single TWR that maximizes the achievable-rate of the network is selected to participate in the communication process. Assuming that the optical channel is affected by path loss, misalignment errors, and atmospheric turbulence, we derive exact and asymptotic closed form expressions for the achievable-rate and the relay with the maximum achievable-rate is selected for communication.
\end{abstract}

Index Terms-Achievable-rate, free-space optics, GammaGamma distribution, pointing error, relay selection, two-way relay.

\section{INTRODUCTION}

$\mathbf{F}$ REE-SPACE OPTICS (FSO) is a line-of-sight (LOS) technology being used for a variety of applications in the telecommunications field. However, the FSO signal undergoes severe fading due to the turbulent atmospheric medium.

Relay-assisted FSO transmission [1], which was introduced in context of the radio frequency (RF) cooperative relaying protocols, has been popularly used as a fading mitigation tool. In the cooperative RF systems, the source broadcasts information to multiple relays, which forward the information to the destination. However, unlike the RF counterpart, the FSO systems are non-broadcasting in nature. Hence, the source needs to be equipped with a multi-laser transmitter for each relay node, with the total source power divided amongst the multiple source-relay links. Furthermore, at high data rates the synchronization of different signals arriving from various relays is cumbersome. Therefore, for networks with power and coverage limitations, selective relaying (SR) based systems gain attention [2], [3]. In these networks a single (best) relay is selected to participate in the communication process.

In the aforementioned SR based systems, the analysis was limited to the outage and error performance for one-way relaying (OWR), where by replacing OWR with two-way relay (TWR), the spectral efficiency of the system can be improved [4]. For selecting a single TWR, the end-to-end instantaneous signal-to-noise ratio (SNR) can be maximized. However, TWR

Manuscript received March 31, 2015; accepted May 31, 2015. Date of publication June 9, 2015; date of current version October 9, 2015. The associate editor coordinating the review of this paper and approving it for publication was Z. Ding.

P. Puri, P. Garg, and M. Aggarwal are with Netaji Subhas Institute of Technology, New Delhi 110 078, India (e-mail: parulpuri9@gmail.com; parul_saini@yahoo.co.in; ermonagarg24@gmail.com).

N. D. Chatzidiamantis is with Aristotle University of Thessaloniki, Thessaloniki 54124, Greece (e-mail: nestoras@auth.gr).

G. K. Karagiannidis is with Khalifa University, 127788 Abu Dhabi, UAE, and also with Aristotle University of Thessaloniki, Thessaloniki 54124, Greece (e-mail: geokarag@ieee.org).

Digital Object Identifier 10.1109/LWC.2015.2442988 communication is characterized by the bi-directional rate pairs of the two-phase transmission [5]. For such systems, net achievable-rate is defined, which depends on the individual capacities that are obtained in the two time-phases. Hence, for TWR assisted systems, an achievable-rate based relay selection scheme is more suitable in comparison to SNR based selection. Further, the existing literature on SR considers only the atmospheric turbulence induced scintillation effects without pointing errors. Since, the severity of pointing errors increases in high rise buildings where FSO transceivers are deployed, it is essential to include these impairments for analysis.

Motivated by the aforementioned literature review, in this paper we present an achievable-rate based relay selection scheme for TWR assisted FSO networks. Considering a parallel relayed FSO network and assuming that the optical channel is affected by path loss, misalignment errors, and atmospheric turbulence, we derive exact and asymptotic closed form expressions for the achievable-rate. Further, the relay with the maximum achievable-rate is selected for communication.

\section{SYSTEM MOdel}

We consider a multiple-node FSO network employing subcarrier phase shift keying (PSK) intensity modulation. All the nodes are capable of half-duplex, amplify-and-forward (AF) two-way communication. The network consists of $K+2$ nodes, with each node having $K+1$ receiving and transmitting antenna apertures. The two nodes selected for data exchange (assuming node selection is done at higher layers), are the source nodes, denoted as $T_{A}$ and $T_{B}$, while the remaining $K$ nodes are the potential relays denoted by $R_{k}$, where $k \in 1,2, \ldots K$.

The system performance is limited by shot noise, $n$, which is modeled as zero mean additive white Gaussian noise (AWGN) with variance $\sigma_{n}^{2}=N_{0} / 2$. It is assumed that the channel state information (CSI), given as $H_{x y}$, where $\{x, y\} \in\left\{A, B, R_{k}\right\}$, of all FSO links is available at $T_{A}$ and $T_{B}$ in the beginning of the communication process. Based on this CSI, the relay that maximizes the net achievable-rate is selected for communication. The selected relay serves as a TWR between $T_{A}$ and $T_{B}$ and carries out the data exchange using two-time phase $\mathrm{AF}$ transmission protocol.

In the first phase, known as the multiple access channel (MAC) phase, both the source terminals $T_{A}$ and $T_{B}$, transmit their information bearing signals, $x_{A}$ and $x_{B}$, respectively, to the relay. The signals received at the relay are

$$
y_{\overrightarrow{A R}}=\Re_{A R} H_{A R} x_{A}+n_{A R} \text { and } y_{\overrightarrow{B R}}=\Re_{B R} H_{B R} x_{B}+n_{B R},
$$

where $\Re=\eta q / h f$ is the responsivity of the photodetector. Here, $\eta$ is the quantum efficiency of the photodetector, $q$ is the electron's charge, $h$ is the Planck's constant, and $f$ is the optical frequency.

In the second phase, known as the broadcast (BC) phase, the relay amplifies the incoming signal and retransmits the 
amplified signal received from $T_{A}\left(T_{B}\right)$ to $T_{B}\left(T_{A}\right)$. The signals received at $T_{A}$ and $T_{B}$ from relay are

$$
\begin{aligned}
& y_{A}=\Re_{R A} H_{R A} G_{R A}\left(\Re_{B R} H_{B R} x_{B}+n_{B R}\right)+n_{R A}, \\
& y_{B}=\Re_{R B} H_{R B} G_{R B}\left(\Re_{A R} H_{A R} x_{A}+n_{A R}\right)+n_{R B},
\end{aligned}
$$

where $G_{R A}$ and $G_{R B}$ denote the relay gains for $R \rightarrow T_{A}$ and $R \rightarrow T_{B}$ transmissions, respectively.

Further, the CSI is modeled as $H=H_{l} \times H_{a} \times H_{p}$, where $H_{l}$ accounts for path loss, $H_{a}$ represents the atmospheric turbulence-induced fading, modeled using the Gamma-Gamma distribution, and $H_{p}$ represents the misalignment fading (pointing errors). All the optical links are assumed to be quasistatic for two time-phases with independent and non-identically distributed (i.n.i.d.) fading statistics. The corresponding probability density function (pdf) of the considered channel model is given by

$f_{H}(h)=\frac{\alpha \beta \xi^{2}}{A_{0} H_{l} \Gamma(\alpha) \Gamma(\beta)} G_{1,3}^{3,0}\left(\left.\frac{\alpha \beta h}{A_{0} H_{l}}\right|_{\xi^{2}-1, \alpha-1, \beta-1} \begin{array}{c}\xi^{2} \\ \xi^{2},\end{array}\right.$

where $\xi=w_{e} / 2 \sigma_{s}, w_{e}$ is the equivalent beam-width radius, $\sigma_{s}$ is the standard deviation of the pointing error displacement at the receiver, $A_{0}$ depends on aperture radius, $r$, and the beam-width radius, $w_{L}$. Further, $\Gamma(\cdot)$ is the Gamma function, $G_{p, q}^{m, n}\left(x \mid \begin{array}{l}a_{1}, \ldots, a_{p} \\ b_{1}, \ldots, b_{q}\end{array}\right)$ is the Meijer's G-function [6, (8.2.1.1)], and $\alpha$ and $\beta$ are the large-scale and small-scale scintillation parameters, respectively, which depend on the Rytov variance $\sigma_{R}^{2}=1.23 C_{n}^{2} k^{7 / 6} L^{11 / 6}$. Here, $k$ is the optical wave number, $C_{n}^{2}$ is the refractive index structure constant, and $L$ is the link distance.

The instantaneous SNR, $\Gamma_{x y}$, of the link between nodes $x$ and $y$, where $\{x, y\} \in\left\{A, B, R_{k}\right\}$, is $\Gamma_{x y}=\frac{E_{s} \eta^{2} P_{x}^{2} H_{x y}^{2}}{\sigma_{n, y}^{2}}$, where $E_{s}$ is the energy of the transmitted symbol, $P_{x}$ is the transmitted optical power of node $x$, and $\sigma_{n, y}^{2}$ is the variance of the noise power at node $y$.

The pdf of $\Gamma_{x y}$ can be obtained by applying transformation of random variables (RVs) in (3) as

$$
f_{\Gamma_{x y}}(\gamma)=\frac{\xi_{x y}^{2}}{2 \gamma \Gamma\left(\alpha_{x y}\right) \Gamma\left(\beta_{x y}\right)} G_{1,3}^{3,0}\left(\alpha_{x y} \beta_{x y} \sqrt{\frac{\gamma}{\bar{\gamma}_{x y}}} \mid \begin{array}{c}
\xi_{x y}^{2}+1 \\
\xi_{x y}^{2}, \alpha_{x y}, \beta_{x y}
\end{array}\right),
$$

where $\bar{\gamma}_{x y}=\frac{E_{s} \eta^{2} P_{x}^{2} H_{l}^{2} A_{0}^{2}}{\sigma_{n, y}^{2}}$ is the average electrical SNR.

\section{Relay SElEction}

For a system with $K$ available relaying nodes, the relay which maximizes the net achievable-rate is selected for the transmission process. The relay selection criterion is given as

$$
R^{*}=\arg \max _{k \in K}\left\{\mathcal{R}_{\text {Sum }, k}^{A F}\right\},
$$

where $\mathcal{R}_{\text {Sum, }}^{A F}$ is the achievable-rate of the $k$ th TWR and can be written as $\mathcal{R}_{\text {Sum }, k}^{A F}=\mathcal{C}_{\overrightarrow{A F}}+\mathcal{C}_{\overleftarrow{A F}}$, where $\mathcal{C}_{\overrightarrow{A F}}$ and $\mathcal{C}_{\overleftarrow{A F}}$ are the ergodic capacities, when information flows from $T_{A} \rightarrow R \rightarrow$ $T_{B}$ and $T_{A} \leftarrow R \leftarrow T_{B}$.
Theorem 1: The ergodic achievable-rate of $\mathrm{AF}$ scheme, $\mathcal{R}_{\text {Sum }}^{A F}$, is given as

$$
\begin{aligned}
& \mathcal{R}_{\text {Sum }}^{A F}=\frac{\xi_{A R}^{2} \xi_{B R}^{2} 2^{\alpha_{A R}+\beta_{A R}+\alpha_{B R}+\beta_{B R}-2}}{32 \pi^{2} \ln (2) \Gamma\left(\alpha_{A R}\right) \Gamma\left(\beta_{A R}\right) \Gamma\left(\alpha_{B R}\right) \Gamma\left(\beta_{B R}\right)} \\
& \times\left\{G_{9,9}^{7,7}\left(\frac{\psi_{R B} \bar{\gamma}_{A R}}{\psi_{A R} \bar{\gamma}_{R B}} \mid \begin{array}{c}
\kappa_{5}, 1, \kappa_{3} \\
\kappa_{4}, \kappa_{6}
\end{array}\right)\right. \\
& +G_{9,9}^{7,7}\left(\frac{\psi_{B R} \bar{\gamma}_{R A}}{\psi_{R A} \bar{\gamma}_{B R}} \mid \begin{array}{c}
\kappa_{7}, 1, \kappa_{1} \\
\kappa_{2}, \kappa_{8}
\end{array}\right) \\
& -S\left[\frac{\psi_{A R}}{\bar{\gamma}_{A R}}, \frac{\psi_{R B}}{\bar{\gamma}_{R B}}\left|\left[\begin{array}{c}
1,0 \\
1,0
\end{array}\right] \begin{array}{c}
0 \\
-
\end{array}\right|\right.
\end{aligned}
$$

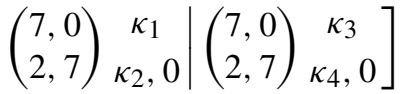

$$
\begin{aligned}
& -S\left[\frac{\psi_{B R}}{\bar{\gamma}_{B R}}, \frac{\psi_{R A}}{\bar{\gamma}_{R A}}\left|\left[\begin{array}{l}
1,0 \\
1,0
\end{array}\right]-\right|\right. \\
& \left.\left.\left(\begin{array}{c}
7,0 \\
2,7
\end{array}\right) \kappa_{4}, 0 \mid\left(\begin{array}{c}
7,0 \\
2,7
\end{array}\right) \kappa_{1}, 0\right]\right\} \text {, }
\end{aligned}
$$

where $\psi_{A R}=\left\{\frac{\alpha_{A R}^{2} \beta_{A R}^{2}}{16}\right\}, \psi_{B R}=\left\{\frac{\alpha_{B R}^{2} \beta_{B R}^{2}}{16}\right\}, \kappa_{1}=\left\{\frac{\xi_{A R}^{2}+1}{2}, \frac{\xi_{A R}^{2}+2}{2}\right\}$, $\kappa_{2}=\left\{\frac{\xi_{A R}^{2}}{2}, \frac{\xi_{A R}^{2}+1}{2}, \frac{\alpha_{A R}}{2}, \frac{\alpha_{A R}+1}{2}, \frac{\beta_{A R}}{2}, \frac{\beta_{A R}+1}{2}\right\}, \kappa_{3}=\left\{\frac{\xi_{B R}^{2}+1}{2}, \frac{\xi_{B R}^{2}+2}{2}\right\}$, $\kappa_{4}=\left\{\frac{\xi_{B R}^{2}}{2}, \frac{\xi_{B R}^{2}+1}{2}, \frac{\alpha_{B R}}{2}, \frac{\alpha_{B R}+1}{2}, \frac{\beta_{B R}}{2}, \frac{\beta_{B R}+1}{2}\right\}, \kappa_{5}=\left\{\frac{2-\xi_{A R}^{2}}{2}, \frac{1-\xi_{A R}^{2}}{2}\right.$, $\left.\frac{2-\alpha_{A R}}{2}, \frac{1-\alpha_{A R}}{2}, \frac{2-\beta_{A R}}{2}, \frac{1-\beta_{A R}}{2}\right\}, \quad \kappa_{6}=\left\{0, \frac{1-\xi_{A R}^{2}}{2},-\frac{\xi_{A R}^{2}}{2}\right\}, \quad \kappa_{7}=$ $\left\{\frac{2-\xi_{B R}^{2}}{2}, \frac{1-\xi_{B R}^{2}}{2}, \frac{2-\alpha_{B R}}{2}, \frac{1-\alpha_{B R}}{2}, \frac{2-\beta_{B R}}{2}, \frac{1-\beta_{B R}}{2}\right\}, \kappa_{8}=\left\{0, \frac{1-\xi_{B R}^{2}}{2}\right.$, $\left.-\frac{\xi_{B R}^{2}}{2}\right\}$, and $S[\cdot]$ is the generalized Meijer's-G function of two variables [7, (1)]. The $S[\cdot]$ function is an extension of the Meijer's-G function and is used to represent the product of three Meijer's-G functions in a closed form.

Proof: A detailed proof is provided in Appendix A.

Note, that function $S[\cdot]$ is not available in standard packages such as MATLAB and Mathematica. However, it can be efficiently evaluated using a MATLAB function, proposed in [5].

Further, substituting (6) in (5) the relay selection is carried out.

\section{ASYMPTOTIC ANALYSIS}

To gain a physical insight of the derived formulae we perform asymptotic analysis at high-SNRs. Assuming i.n.i.d. fading statistics, the cumulative distribution function (cdf) of the instantaneous SNR between the terminal nodes at high SNRs can be written as [8]

$$
\mathcal{F}_{\Gamma_{A B}}(\gamma) \approx 1-\operatorname{Pr}\left(\Gamma_{A R}>\gamma\right) \operatorname{Pr}\left(\Gamma_{B R}>\gamma\right),
$$

where $\mathcal{F}_{\Gamma_{A B}}(\cdot)$ represents the cdf. Differentiating (7), the pdf of $\Gamma_{A B}$ can be written as

$$
\begin{aligned}
f_{\Gamma_{A B}}(\gamma) \approx f_{\Gamma_{A R}}(\gamma)+f_{\Gamma_{R B}}(\gamma)-\mathcal{F}_{\Gamma_{A R}}(\gamma) f_{\Gamma_{R B}}(\gamma) & \\
& -f_{\Gamma_{A R}}(\gamma) \mathcal{F}_{\Gamma_{R B}}(\gamma) .
\end{aligned}
$$

The last two terms in (8) may be neglected at high SNRs. Now, using the definition of ergodic capacity given as $\mathcal{C}_{A B}=$ $\frac{1}{\ln (2)} \int_{0}^{\infty} \ln (1+\gamma) f_{\Gamma_{A B}}(\gamma) d \gamma$, substituting the pdf using (4) and (8), expressing the logarithmic integrand in terms of the Meijer's-G function [6, (8.4.6.5)], and integrating using $[6,(2.24 .1 .1)]$, an analytical expression for the ergodic capacity in terms of the Meijer's-G function can be obtained. Using the series expansion of the Meijer's-G function, for the condition 


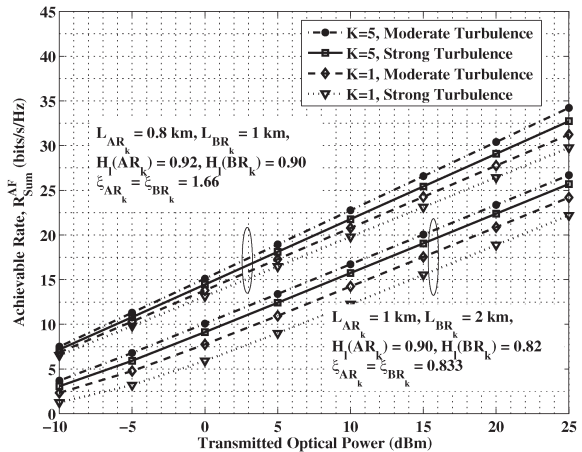

Fig. 1. Achievable-rate vs. transmitted optical power plot for TWR-FSO network having $K+2$ nodes, for strong and moderate atmospheric turbulence with varying pointing errors and path loss.

$\lim _{z \rightarrow 0}{ }_{2} F_{3}(a ; b ; z)=1$, where ${ }_{p} F_{q}(a ; b ; z)$ is the hypergeometric function, the expression for asymptotic achievable-rate can be written as

$$
\begin{aligned}
\mathcal{R}_{\text {Sum }}^{A F} \approx & {\left[\frac{\xi_{A R}^{2} 2^{\alpha_{A R}+\beta_{A R}-2}}{2 \pi \ln (2) \Gamma\left(\alpha_{A R}\right) \Gamma\left(\beta_{A R}\right)}\right.} \\
& \times \sum_{k=1}^{8} \frac{\prod_{\substack{j=1 \\
j \neq k}}^{8} \Gamma\left(\kappa_{10}(j)-\kappa_{10}(k)\right) \Gamma\left(1+\kappa_{10}(k)\right)}{\prod_{j=1}^{3} \Gamma\left(\kappa_{9}(j)-\kappa_{10}(k)\right)} \\
& \left.\times\left(\frac{\psi_{A R}}{\bar{\gamma}_{A R}}\right)^{\kappa_{10}(k)}\right] \\
+ & {\left[\frac{\xi_{B R}^{2} 2^{\alpha_{B R}+\beta_{B R}-2}}{2 \pi \ln (2) \Gamma\left(\alpha_{B R}\right) \Gamma\left(\beta_{B R}\right)}\right.} \\
& \times \sum_{k=1}^{8} \frac{\prod_{\substack{j=1 \\
j \neq k}}^{8} \Gamma\left(\kappa_{12}(j)-\kappa_{12}(k)\right) \Gamma\left(1+\kappa_{12}(k)\right)}{\prod_{j=1}^{3} \Gamma\left(\kappa_{11}(j)-\kappa_{12}(k)\right)} \\
& \left.\times\left(\frac{\psi_{B R}}{\bar{\gamma}_{B R}}\right)^{\kappa_{12}(k)}\right],
\end{aligned}
$$

where $\kappa_{9}=\left\{1, \kappa_{1}\right\}, \kappa_{10}=\left\{\kappa_{2}, 0,0\right\}, \kappa_{11}=\left\{1, \kappa_{3}\right\}$, and $\kappa_{12}=$ $\left\{\kappa_{4}, 0,0\right\}$. The asymptotic expression in (9) is dominated by $\min \left\{\alpha_{x y}, \beta_{x y}, \xi_{x y}, 1\right\}$.

\section{NUMERICAL RESULTS AND DISCUSSION}

The analysis and simulations are done under moderate $\left(C_{n}^{2}=3 \times 10^{-14} \mathrm{~m}^{-2 / 3}\right)$ and strong $\left(C_{n}^{2}=1 \times 10^{-13} \mathrm{~m}^{-2 / 3}\right)$ atmospheric turbulence conditions. The aperture radius, $r=0.1$ $\mathrm{m}$ and laser wavelength, $\lambda=1.55 \times 10^{-6} \mathrm{~m}$. It is assumed the transmit powers of all nodes is same, $P_{A}=P_{B}=P_{R}=P_{S}$, and the relay transmits with equal power to both the source nodes in $\mathrm{BC}$ phase.

Fig. 1 is a plot of the achievable-rate with respect to $P_{S}$, for a TWR-FSO network having $K+2$ nodes. The plot shows a comparison of achievable-rates when there is no relay selection, i.e. $K=1$, and when one node out of $K=5$ nodes is selected to establish communication between $T_{A}$ and $T_{B}$. All the links are i.n.i.d. with $L_{A R_{k}}=0.8 \mathrm{~km}, L_{B R_{k}}=1 \mathrm{~km}$ and $L_{A R_{k}}=1 \mathrm{~km}$, $L_{B R_{k}}=2 \mathrm{~km}, k \in\{1,2, \ldots K\}$. As observed, for both strong and moderate turbulence, a higher achievable-rate is achieved when relay selection is carried out.

In Fig. 2, we consider a TWR-FSO link with two terminal nodes, $T_{A}$ and $T_{B}$, and a single relay, $R$. For the considered link, we plot the achievable-rate with respect to the ratio of

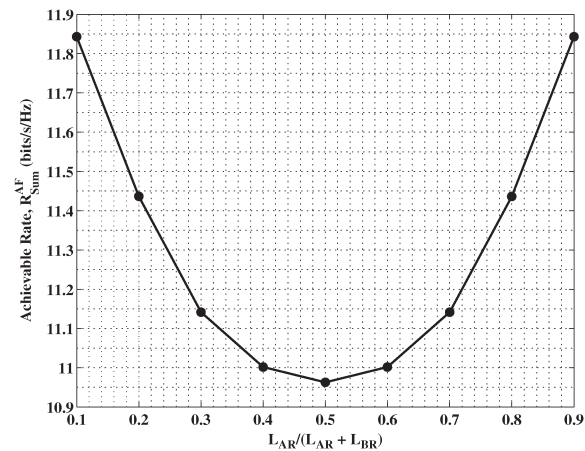

Fig. 2. Achievable-rate vs. ratio of distance, $L_{A R} /\left(L_{A R}+L_{B R}\right)$, plot for TWR-FSO link

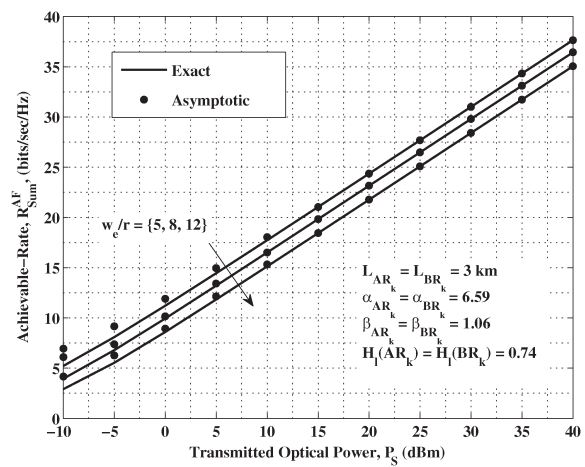

Fig. 3. Exact and asymptotic achievable-rate vs. transmitted optical power plot for $K+2=7$ nodes TWR-FSO network for varying values of $w_{e} / r$.

distance between $T_{A}-R$ and total distance between $T_{A}-T_{B}$, when $L_{A B}=4 \mathrm{~km}, P_{A}=P_{B}=P_{R}=5 \mathrm{dBm}, w_{e} / r=10$, and $\sigma_{s} / r=3$. As seen from the plot, the $R_{S u m}^{A F}$ is maximum when the relay is closer to one of the terminals. A sudden dip in achievable-rate is observed when the length ratio is between 0.25 and 0.75 . Using this result, it can be established that it is more likely to select a relay that is closer to either one of the terminal nodes, since it has a higher achievable-rate.

Fig. 3 is a plot of the exact and asymptotic achievable-rate with respect to $P_{S}$, when $K+2=7, L_{A R_{k}}=L_{B R_{k}}=3 \mathrm{~km}$, $\sigma_{s} / r=5$ and with varying values of $w_{e} / r$. As seen from this plot, the achievable-rate decreases as the equivalent beam-width radius is increased. Also, it is evident the asymptotic curves converge quite fast with the exact curves, thereby demonstrating the tightness of the asymptotic expressions at high-SNRs.

\section{CONCLUSION}

We presented an achievable-rate based relay selection scheme for a multiple node FSO network. Closed form exact and asymptotic expressions for the achievable-rate were derived. The impact of various parameters such as link lengths, turbulence strength, beam-width radius, jitter variance, and transmitted power on the system performance was demonstrated.

\section{APPENDIX A}

The ergodic capacity achieved when information flows from $T_{A} \rightarrow R \rightarrow T_{B}$ is given as [9]

$$
\mathcal{C}_{\overrightarrow{A F}}=\frac{1}{2 \ln 2} \int_{0}^{\infty} \frac{1}{s}\left(1-e^{-s}\right) \mathcal{M}_{\frac{1}{\Gamma_{A B}}}(s) d s,
$$

where $1 / 2$ is due to half-duplex relaying and $\mathcal{M}_{\frac{1}{\Gamma_{A B}}}(s)$ is the moment generating function (mgf) of the inverse of the 
equivalent end-to-end SNR, $\Gamma_{A B}$. When the relay gain is set as $G_{R B}^{2}=\left(H_{A R}^{2} C_{1}\right)^{-1}+C_{2} N_{0}, \Gamma_{A B}$ can be written in terms of the instantaneous SNRs between the terminal nodes and relay as

$$
\Gamma_{A B}=\frac{\Gamma_{A R} \Gamma_{R B}}{C_{2}+C_{1} \Gamma_{A R}+\Gamma_{R B}} .
$$

For CSI-assisted relays, when $C_{1}=1$ and $C_{2}=0,(\mathrm{~A} .2)$ can be re-written as $\Gamma_{A B}=\left(\frac{1}{\Gamma_{A R}}+\frac{1}{\Gamma_{R B}}\right)^{-1}$.

Since, $\Gamma_{A R}$ and $\Gamma_{R B}$ are statistically independent RVs, we obtain

$$
\mathcal{M}_{\frac{1}{\Gamma_{A B}}}(s)=\mathcal{M}_{\frac{1}{\Gamma_{A R}}}(s) \times \mathcal{M}_{\frac{1}{\Gamma_{R B}}}(s)
$$

where $\mathcal{M}_{\frac{1}{\Gamma_{A R}}}(s)$ and $\mathcal{M}_{\frac{1}{\Gamma_{R B}}}(s)$ denote the mgfs of the reciprocals of $\Gamma_{A R}$ and $\Gamma_{R B}$, respectively. The mgf of reciprocal of $\Gamma_{x y}$, where $\{x y\} \in\{A R, R B\}$, is given as

$$
\mathcal{M}_{\frac{1}{\Gamma_{x y}}}(s)=\int_{0}^{\infty} \exp \left(-\frac{s}{\Gamma_{x y}}\right) f_{\Gamma_{x y}}(\gamma) d \gamma .
$$

Substituting (4) and expressing the exponential term in terms of Meijer's-G function [6, (8.4.11.1)], (A.4) can be integrated using $[6,(2.24 .1 .1)]$ as

$$
\begin{aligned}
& \mathcal{M}_{\frac{1}{\Gamma_{x y}}}(s)=\frac{\xi_{x y}^{2} 2^{\alpha_{x y}+\beta_{x y}-1}}{4 \pi \Gamma\left(\alpha_{x y}\right) \Gamma\left(\beta_{x y}\right)} \\
& \quad \times G_{2,7}^{7,0}\left(\left.\frac{\alpha_{x y}^{2} \beta_{x y}^{2} s}{16 \bar{\gamma}_{x y}}\right|_{\frac{\xi_{x y}}{2}, \frac{\xi_{x y}{ }^{2}+1}{2}, \frac{\alpha_{x y}}{2}, \frac{\alpha_{x y}+1}{2}, \frac{\beta_{x y}}{2}, \frac{\beta_{x y}+1}{2}, 0}\right) .
\end{aligned}
$$

Now, substituting (A.3) in (A.1), the ergodic capacity can be re-written as

$$
\begin{aligned}
\mathcal{C}_{\overrightarrow{A F}}=\frac{1}{2 \log (2)} \underbrace{\int_{0}^{\infty} s^{-1} \mathcal{M}_{\frac{1}{\Gamma_{A R}}}(s) \mathcal{M}_{\frac{1}{\Gamma_{R B}}}(s) d s}_{I_{1}} \\
-\underbrace{\int_{0}^{\infty} e^{-s} s^{-1} \mathcal{M}_{\frac{1}{\Gamma_{A R}}}(s) \mathcal{M}_{\frac{1}{\Gamma_{R B}}}(s) d s}_{I_{2}}
\end{aligned}
$$

To evaluate (A.6), we consider the integrals $I_{1}$ and $I_{2}$ separately. Substituting (A.5), $I_{1}$ can be re-written as

$$
\begin{aligned}
& I_{1}=\frac{\xi_{A R}^{2} \xi_{B R}^{2} 2^{\alpha_{A R}+\beta_{A R}+\alpha_{B R}+\beta_{B R}-2}}{16 \pi^{2} \Gamma\left(\alpha_{A R}\right) \Gamma\left(\beta_{A R}\right) \Gamma\left(\alpha_{B R}\right) \Gamma\left(\beta_{B R}\right)} \\
& \times \int_{0}^{\infty} \frac{1}{s} G_{2,7}^{7,0}\left(\frac{s \psi_{A R}}{\bar{\gamma}_{A R}} \mid \begin{array}{c}
\kappa_{1} \\
\kappa_{2}, 0
\end{array}\right) G_{2,7}^{7,0}\left(\frac{s \psi_{R B}}{\bar{\gamma}_{R B}} \mid \begin{array}{c}
\kappa_{3} \\
\kappa_{4}, 0
\end{array}\right) d s .
\end{aligned}
$$

Integrating (A.7) using [6, (2.24.1.1)], we obtain

$$
\begin{aligned}
& I_{1}=\frac{\xi_{A R}^{2} \xi_{B R}^{2} 2^{\alpha_{A R}+\beta_{A R}+\alpha_{B R}+\beta_{B R}-2}}{16 \pi^{2} \Gamma\left(\alpha_{A R}\right) \Gamma\left(\beta_{A R}\right) \Gamma\left(\alpha_{B R}\right) \Gamma\left(\beta_{B R}\right)} \\
& \times G_{9,9}^{7,7}\left(\frac{\psi_{R B} \bar{\gamma}_{A R}}{\psi_{A R} \bar{\gamma}_{R B}} \mid \begin{array}{c}
\kappa_{5}, 1, \kappa_{3} \\
\kappa_{4}, \kappa_{6}
\end{array}\right) .
\end{aligned}
$$

Further, expressing the exponential term in $I_{2}$ in terms of Meijer's-G function [6, (8.4.11.1)], substituting (A.5), and ex- pressing $I_{2}$ in terms of integral using [6, (8.2.1.1)], we obtain

$$
\begin{aligned}
I_{2}= & \frac{\xi_{A R}^{2} \xi_{B R}^{2} 2^{\alpha_{A R}+\beta_{A R}+\alpha_{B R}+\beta_{B R}-2}}{16 \pi^{2} \Gamma\left(\alpha_{A R}\right) \Gamma\left(\beta_{A R}\right) \Gamma\left(\alpha_{B R}\right) \Gamma\left(\beta_{B R}\right)} \frac{1}{(2 \pi i)^{2}} \int_{L_{1}} \int_{L_{2}} \\
\times & {\left[\frac{\Gamma(-x) \Gamma(-y) \prod_{j=1}^{6} \Gamma\left(\kappa_{2}(j)-x\right) \prod_{j=1}^{6} \Gamma\left(\kappa_{4}(j)-y\right)}{\prod_{j=1}^{2} \Gamma\left(\kappa_{1}(j)-x\right) \prod_{j=1}^{2} \Gamma\left(\kappa_{3}(j)-y\right)}\right.} \\
& \left.\times\left(\frac{\psi_{A R}}{\bar{\gamma}_{A R}}\right)^{x}\left(\frac{\psi_{B R}}{\bar{\gamma}_{B R}}\right)^{y} \int_{0}^{\infty} s^{x+y-1} G_{0,1}^{1,0}(s \mid-) d s\right] d x d y .
\end{aligned}
$$

Now, using [7, (1)], (A.9) can be expressed in terms of the generalized function of two variables as

$$
\begin{aligned}
& I_{2}=\frac{\xi_{A R}^{2} \xi_{B R}^{2} 2^{\alpha_{A R}+\beta_{A R}+\alpha_{B R}+\beta_{B R}-2}}{16 \pi^{2} \Gamma\left(\alpha_{A R}\right) \Gamma\left(\beta_{A R}\right) \Gamma\left(\alpha_{B R}\right) \Gamma\left(\beta_{B R}\right)} \\
& \times S\left[\frac{\psi_{A R}}{\bar{\gamma}_{A R}}, \frac{\psi_{R B}}{\bar{\gamma}_{R B}}\left|\left[\begin{array}{l}
1,0 \\
1,0
\end{array}\right]-\right| \begin{array}{c}
0 \\
-\mid \\
2,7
\end{array}\right) \kappa_{2}, 0 \mid \\
& \left.\left(\begin{array}{c}
7,0 \\
2,7
\end{array}\right) \kappa_{3}, 0\right] \text {. }
\end{aligned}
$$

Substituting (A.8) and (A.10) in (A.7), the ergodic capacity, $\mathcal{C}_{\overrightarrow{A F}}$ is obtained as

$$
\begin{aligned}
& \mathcal{C}_{\overrightarrow{A F}}= \frac{\xi_{A R}^{2} \xi_{B R}^{2} 2^{\alpha_{A R}+\beta_{A R}+\alpha_{B R}+\beta_{B R}-2}}{32 \pi^{2} \ln (2) \Gamma\left(\alpha_{A R}\right) \Gamma\left(\beta_{A R}\right) \Gamma\left(\alpha_{B R}\right) \Gamma\left(\beta_{B R}\right)} \\
& \times\left\{G_{9,9}^{7,7}\left(\frac{\psi_{R B} \bar{\gamma}_{A R}}{\psi_{A R} \bar{\gamma}_{R B}} \mid \begin{array}{c}
\kappa_{5}, 1, \kappa_{3} \\
\kappa_{4}, \kappa_{6}
\end{array}\right)\right. \\
&\left.\quad-S\left[\begin{array}{l}
\left.\frac{\psi_{A R}}{\bar{\gamma}_{A R}}, \frac{\psi_{B R}}{\bar{\gamma}_{B R}}\left|\left[\begin{array}{c}
1,0 \\
1,0
\end{array}\right]-\right| \begin{array}{c}
7,0 \\
2,7
\end{array}\right) \kappa_{1}, 0 \mid \\
\left(\begin{array}{c}
7,0 \\
2,7
\end{array}\right) \kappa_{3}, 0
\end{array}\right]\right\} .
\end{aligned}
$$

Using the same approach, we can obtain the ergodic capacity, $\mathcal{C}_{\overleftarrow{A F}}$. Using $\mathcal{C}_{\overrightarrow{A F}}$ and $\mathcal{C}_{\overleftarrow{A F}}$, the achievable-rate for a TWR-FSO link using single AF relay is obtained as in (6).

\section{REFERENCES}

[1] M. Safari and M. Uysal, "Relay-assisted free-space optical communication," IEEE Trans. Wireless Commun., vol. 7, no. 12, pp. 5441-5449, Dec. 2008.

[2] N. D. Chatzidiamantis, D. S. Michalopoulos, E. E. Kriezis, G. K. Karagiannidis, and R. Schober, "Relay selection protocols for relay-assisted free-space optical systems," IEEE/OSA J. Opt. Commun. Netw., vol. 5, no. 1, pp. 92-103, Jan. 2013.

[3] M. R. Bhatnagar, "Average BER analysis of relay selection based decode-and-forward cooperative communication over Gamma-Gamma fading FSO links," in Proc. IEEE ICC, Budapest, Hungary, Jun. 2013, pp. 3142-3147.

[4] P. Puri, P. Garg, and M. Aggarwal, "Outage and error rate analysis of network-coded coherent TWR-FSO systems," IEEE Photon. Tech. Lett., vol. 26, no. 18, pp. 1797-1800, Sep. 2014.

[5] P. Puri, N. D. Chatzidiamantis, P. Garg, M. Aggarwal, and G. K. Karagiannidis, "Maximizing the achievable sum-rate of FSO networks via two-way relay selection," submitted for publication.

[6] A. P. Prudnikov, Y. A. Brychkov, and O. I. Marichev, Integrals and Series Volume 3: More Special Functions, 1st Ed. New York, NY, USA: Gordon and Breach, 1986.

[7] B. L. Sharma and R. F. A. Abiodun, "Generating function for generalized function of two variables," Proc. Amer. Math. Soc., vol. 46, no. 1, pp. 69-72, Oct. 1974.

[8] S. Ikki and M. H. Ahmed, "Performance analysis of cooperative diversity wireless networks over Nakagami-m fading channel," IEEE Commun. Lett., vol. 11, no. 4, pp. 334-336, Apr. 2007.

[9] F. Yilmaz, O. Kucur, and M.-S. Alouini, "Exact capacity analysis of multihop transmission over amplify-and-forward relay fading channels," in Proc. IEEE Int. Symp. PIMRC, Istanbul, Turkey, 2010, pp. 2293-2298. 